\title{
Black holes by analytic continuation
}

\author{
D. Amati \\ SISSA, Trieste, Italy \\ and INFN, Sezione di Trieste, Trieste, Italy \\ J. G. Russo \\ Theory Division, CERN, CH-1211 Geneva 23, Switzerland \\ (Received 18 October 1996)
}

\begin{abstract}
In the context of a two-dimensional exactly solvable model, the dynamics of quantum black holes is obtained by analytically continuing the description of the regime where no black hole is formed. The resulting spectrum of outgoing radiation departs from the one predicted by the Hawking model in the region where the outgoing modes arise from the horizon with Planck-order frequencies. This occurs early in the evaporation process, and the resulting physical picture is unconventional. The theory predicts that black holes will only radiate out an energy of Planck mass order, stabilizing after a transitory period. The continuation from a regime without black hole formation-accessible in the $1+1$ gravity theory considered-is implicit in an $S$-matrix approach and suggests in this way a possible solution to the problem of information loss. [S0556-2821(97)00612-7]

PACS number(s): 04.70.Dy, 04.60.Kz
\end{abstract}

\section{INTRODUCTION}

It has often been advocated [1,2] that the study of scattering of matter and radiation in a quantum gravity theory should solve the conflict between classical black-hole solutions and quantum mechanics, which leads to information loss [3]. The mere existence of an $S$ matrix below the threshold of black hole formation would be enough to exhibit, through its analytic structure, eventual thresholds for the creation of new objects and to describe, through analytic continuation, the physics above them in a unitary framework.

By studying a semiclassical solvable model in which the black-hole evolution can be explicitly investigated, we will see that analytic continuation (from below the threshold of black-hole formation to above it) completely determines the structure of the theory in the regime in which black holes are formed. The model is the two-dimensional dilaton gravity with matter [Russo-Susskind-Thorlacius (RST) [4]], which represents a toy model for spherically symmetric infalling shells in four-dimensional gravity. Because of quantum effects there is a threshold on the incident matter energy density under which there is no black-hole formation.

We shall adopt the usual boundary conditions below the threshold, so the subcritical regime will be as in the RST model. It will then be shown that the corresponding outgoing energy-momentum tensor can be straightforwardly continued above the critical incoming energy-density flux. The semiclassical supercritical treatment that would give rise to the same outgoing radiation requires a boundary at the apparent horizon (this is at variance with the standard boundary on the singularity). As a result, a very unconventional picture appears. In particular, Hawking radiation stops early in the evaporation process and a stable macroscopic black hole remains in the final state, thus avoiding the information loss. This goes in the direction advocated by Giddings [5] as a possible solution of the information loss problem.

In Secs. II and III we briefly review the model of [4] and the subcritical regime. The obtained outgoing energymomentum tensor may be continued beyond the thresholdSec. IV - and we discuss in Sec. V which boundary conditions would reproduce it. Section VI generalizes the preceding results to other infalling distributions of interest. In Sec. VII we summarize the physical picture and discuss the origin of the differences with preceding treatments.

\section{SEMICLASSICAL DILATON GRAVITY}

The semiclassical action of the RST model (which includes the one-loop quantum anomaly) is given by

$$
\begin{aligned}
S= & \frac{1}{2 \pi} \int d^{2} x \sqrt{-g}\left(e^{-2 \phi}\left[R+4(\nabla \phi)^{2}+4 \lambda^{2}\right]\right. \\
& \left.-\frac{1}{2} \sum_{i=1}^{N}\left(\nabla f_{i}\right)^{2}-\kappa\left[2 \phi R+R\left(\nabla^{2}\right)^{-1} R\right]\right), \quad \kappa=\frac{N}{48} .
\end{aligned}
$$

In the conformal gauge $g_{ \pm \pm}=0, g_{+-}=-(1 / 2) e^{2 \rho}$, the action is simplified by introducing new fields $\chi, \Omega$, related to $\rho$ and $\phi$ by

$$
\chi=4 \kappa \rho+e^{-2 \phi}-2 \kappa \phi, \quad \Omega=e^{-2 \phi}+2 \kappa \phi .
$$

Then action (2.1) takes the form

$$
\begin{aligned}
S= & \frac{1}{\pi} \int d^{2} x\left(\frac{1}{4 \kappa}\left(-\partial_{+} \chi \partial_{-} \chi+\partial_{+} \Omega \partial_{-} \Omega\right)+\lambda^{2} e^{(1 / 2 \kappa)(\chi-\Omega)}\right. \\
& \left.+\frac{1}{2} \sum_{i=0}^{N} \partial_{+} f_{i} \partial_{-} f_{i}\right)
\end{aligned}
$$

with the constraints [corresponding to the $g_{ \pm \pm}$equations of motion of action (2.1)] 


$$
\begin{aligned}
t_{ \pm}\left(x^{ \pm}\right)= & \frac{1}{4 \kappa}\left(-\partial_{ \pm} \chi \partial_{ \pm} \chi+\partial_{ \pm} \Omega \partial_{ \pm} \Omega\right)+\partial_{ \pm}^{2} \chi \\
& +\frac{1}{2} \sum_{i=0}^{N} \partial_{ \pm} f_{i} \partial_{ \pm} f_{i} .
\end{aligned}
$$

The functions $t_{ \pm}\left(x^{ \pm}\right)$are determined by boundary conditions.

Let us consider a general distribution of incoming matter:

$$
T_{++}\left(x^{+}\right)=\frac{1}{2} \sum_{i=0}^{N} \partial_{+} f_{i} \partial_{+} f_{i} .
$$

In the Kruskal gauge $\chi=\Omega$, the solution to the semiclassical equations of motion and the constraints is given by

$$
\begin{aligned}
\Omega= & \chi=-\lambda^{2} x^{+}\left[x^{-}+\lambda^{-2} P_{+}\left(x^{+}\right)\right]-\kappa \ln \left(-\lambda^{2} x^{+} x^{-}\right) \\
& +\lambda^{-1} M\left(x^{+}\right)
\end{aligned}
$$

where $M\left(x^{+}\right)$and $P_{+}\left(x^{+}\right)$physically represent the total energy and total Kruskal momentum of the incoming matter at advanced time $x^{+}$:

$$
\begin{gathered}
P_{+}\left(x^{+}\right)=\int_{0}^{x^{+}} d x^{+} T_{++}\left(x^{+}\right), \\
M\left(x^{+}\right)=\lambda \int_{0}^{x^{+}} d x^{+} x^{+} T_{++}\left(x^{+}\right) .
\end{gathered}
$$

In the particular case $T_{++}=0$, Eq. (2.5) reduces to the familiar linear dilaton vacuum

$$
e^{-2 \phi}=e^{-2 \rho}=-\lambda^{2} x^{+} x^{-}
$$

In Minkowski coordinates $\sigma^{ \pm}, \lambda x^{ \pm}= \pm e^{ \pm \lambda \sigma^{ \pm}}$, one has $d s^{2}=-d \tau^{2}+d \sigma^{2}, \phi=-\lambda \sigma, \sigma^{ \pm}=\tau \pm \sigma$.

The curvature scalar of the geometry, $R=8 e^{-2 \rho} \partial_{+} \partial_{-} \rho$, can be conveniently written as

$$
R=8 e^{-2 \rho} \frac{1}{\Omega^{\prime}(\phi)}\left(\partial_{+} \partial_{-} \chi-4 \partial_{+} \phi \partial_{-} \phi e^{-2 \phi}\right)
$$

In this form we see that, generically, there will be a curvature singularity at $\phi=\phi_{\mathrm{cr}}=-\frac{1}{2} \ln \kappa$ where $\Omega^{\prime}(\phi)=0$.

As observed in Ref. [4], there are two different regimes, according to whether $T_{++}\left(x^{+}\right)$is smaller or greater than the critical flux:

$$
T_{++}^{\mathrm{cr}}\left(x^{+}\right)=\frac{\kappa}{x^{+2}}
$$

Note that the existence of the threshold is a quantum effect. Indeed, $\kappa$ is proportional to $\hbar$ (here we have set $\hbar=1$ ) and thus $T_{++}^{\text {cr }}$ vanishes as $\hbar \rightarrow 0$. Using Eq. (2.5) it can be seen that the line $\Omega=\Omega_{\mathrm{cr}}\left[\equiv \Omega\left(\phi_{\mathrm{cr}}\right)\right]$ is timelike if $T_{++}\left(x^{+}\right)$ $<T_{++}^{\mathrm{cr}}\left(x^{+}\right)$, and it becomes spacelike as soon as $T_{++}\left(x^{+}\right)$ $>T_{++}^{\mathrm{cr}}\left(x^{+}\right)$.

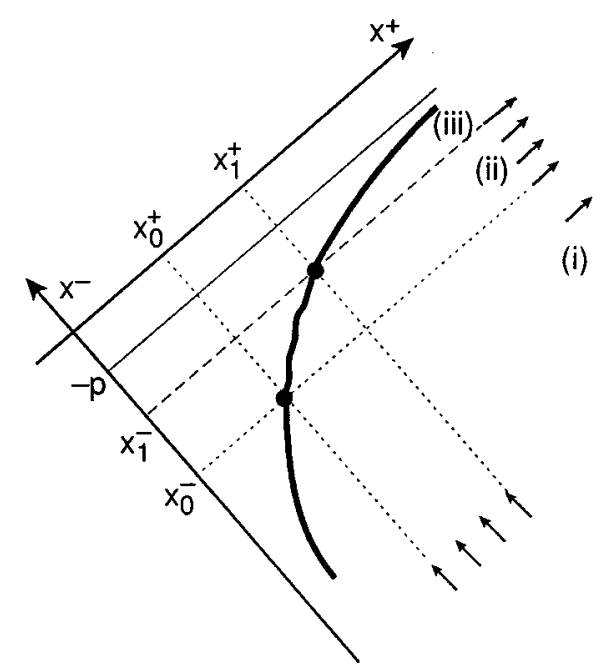

FIG. 1. Kruskal diagram in the subcritical regime.

\section{SUBCRITICAL REGIME}

In order to investigate the analytic continuation of the subcritical regime to a supercritical regime, it is convenient to explore in more detail the subcritical theory of Ref. [4]. Let us assume that the geometry is originally the linear dilaton vacuum, and there is an incoming energy density flux $T_{++}\left(x^{+}\right)<T_{++}^{\mathrm{cr}}\left(x^{+}\right)$, which is different from 0 for $x_{0}^{+}$ $<x^{+}<x_{1}^{+}$. Let us define region (i) as $x^{-}<x_{0}^{-}, x_{0}^{-}=$ $-\kappa /\left(\lambda^{2} x_{0}^{+}\right)$, and region (ii) as that between $x_{0}^{-}$and $x_{1}^{-}$(see Fig. 1).

In region (i), the solution is given by Eq. (2.5), which is completely specified by the asymptotic boundary conditions and by demanding a continuous matching with the linear dilaton vacuum in the infalling line. In region (ii) the boundary $\phi=\phi_{\text {cr }}$ is timelike and boundary conditions are needed in order to determine the evolution. Continuity along the line $x^{-}=x_{0}^{-}$requires that the solution in region (ii) be of the form

$\Omega^{(\mathrm{ii})}\left(x^{+}, x^{-}\right)=\Omega^{(\mathrm{i})}\left(x^{+}, x^{-}\right)+F\left(x^{-}\right), \quad F\left(x_{0}^{-}\right)=0$.

The "reflecting', RST boundary conditions follow from the requirement of finite curvature on the boundary line. Indeed, from Eq. (2.7), we see that in order to have finite curvature at the line $\Omega^{\prime}(\phi)=0$ it is necessary that

$$
\left.\partial_{+} \phi \partial_{-} \phi\right|_{\phi=\phi_{\mathrm{cr}}}=-\frac{\lambda^{2}}{4 \kappa},
$$

where we have used the equation of motion (in the gauge $\chi=\Omega) \partial_{+} \partial_{-} \chi=-\lambda^{2}$. Equation (3.2) implies, in particular [see Eq. (2.2)],

$$
\left.\partial_{+} \Omega\right|_{\phi=\phi_{\mathrm{cr}}}=\left.\partial_{-} \Omega\right|_{\phi=\phi_{\mathrm{cr}}}=0 .
$$

As a result, the function $F\left(x^{-}\right)$is determined to be

$$
F\left(x^{-}\right)=\kappa \ln \left[\frac{x^{-}}{x^{-}+\lambda^{-2} P_{+}\left(\hat{x}^{+}\right)}\right]-\lambda^{-1} M\left(\hat{x}^{+}\right),
$$

where $\hat{x}^{+}=\hat{x}^{+}\left(x^{-}\right)$is the boundary curve given by 


$$
-\lambda^{2} \hat{x}^{+}\left[x^{-}+\lambda^{-2} P_{+}\left(\hat{x}^{+}\right)\right]=\kappa .
$$

Finally, in region (iii), the geometry is matched with the vacuum:

$$
\begin{gathered}
\Omega^{(\mathrm{iii})}=\chi^{(\mathrm{iii})}=-\lambda^{2} x^{+}\left(x^{-}+p\right)-\kappa \ln \left[-\lambda^{2} x^{+}\left(x^{-}+p\right)\right], \\
p \equiv \lambda^{-2} P_{+}\left(x_{1}^{+}\right) .
\end{gathered}
$$

In Minkowski coordinates $\lambda\left(x^{-}+p\right)=-e^{-\lambda \sigma^{-}}, \lambda x^{+}$ $=e^{\lambda \sigma^{+}}$, this simply becomes $d s^{2}=-d \sigma^{+} d \sigma^{-}, \phi=-\lambda \sigma$.

The outgoing energy density fluxes measured by an out observer can be found from the constraints. They are given by

$$
\begin{gathered}
T_{--}^{(\mathrm{i})}\left(x^{-}\right)=\kappa\left[\frac{1}{\left(x^{-}+p\right)^{2}}-\frac{1}{x^{-2}}\right], \\
T_{--}^{(\mathrm{ii})}\left(x^{-}\right)=\kappa \frac{1}{\left(x^{-}+p\right)^{2}}-\frac{\lambda^{4}}{\frac{\kappa}{\hat{x}^{+2}}-T_{++}\left(\hat{x}^{+}\right)}, \\
T_{--}^{(\mathrm{iii})}=0 .
\end{gathered}
$$

The radiation energy emitted between times $\sigma_{1}^{-}$and $\sigma_{2}^{-}$is given by the integral

$$
E=\int_{\sigma_{1}^{-}}^{\sigma_{2}^{-}} d \sigma^{-} T_{\sigma^{-} \sigma^{-}}=-\lambda \int_{x_{1}^{-}}^{x_{2}^{-}} d x^{-}\left(x^{-}+p\right) T_{--} .
$$

Thus the total radiated energies in regions (i) and (ii) are

$$
\begin{aligned}
& E_{\mathrm{out}}^{(\mathrm{i})}=-\lambda \int_{-\infty}^{x_{0}^{-}} d x^{-}\left(x^{-}+p\right) T_{--}^{(\mathrm{i})}=-\frac{\lambda \kappa p}{x_{0}^{-}}-\lambda \kappa \ln \left(1+\frac{p}{x_{0}^{-}}\right) \\
& E_{\mathrm{out}}^{(\mathrm{ii})}=-\lambda \int_{x_{0}^{-}}^{x_{1}^{-}} d x^{-}\left(x^{-}+p\right) T_{--}^{(\mathrm{ii})} \\
&=m+\frac{\lambda \kappa p}{x_{0}^{-}}+\lambda \kappa \ln \left(1+\frac{p}{x_{0}^{-}}\right)
\end{aligned}
$$

where $m \equiv M\left(x_{1}^{+}\right)$is the total Arnowitt-Deser-Misner (ADM) energy of the initial configuration. The coordinate $x_{0}^{-}$is related to the time $x_{0}^{+}$, at which the incoming flux begins, by $x_{0}^{-}=-\left(\kappa / \lambda^{2} x_{0}^{+}\right)$. For $p \ll\left|x_{0}^{-}\right|$("low-energy" fluxes), one has $E_{\text {out }}^{(\mathrm{i})} \ll m, E_{\text {out }}^{\text {(ii) }} \sim m$, that is, most of the energy comes out by pure reflection on the space-time boundary. For $p \cong-x_{0}^{-}, p<\left|x_{0}^{-}\right|$, the logarithm becomes large and negative so that the energy radiated in region (ii) is negative.

Note that it is possible to have $T_{++}>T_{++}^{\mathrm{cr}}=\kappa / x^{+^{2}}$ and yet $p<-x_{0}^{-}$. This means that the threshold given by the singularity of the logarithm in Eqs. (3.10) and (3.11) is not in general the threshold for black-hole formation. To see this explicitly, let us consider the simplest case in which the incoming energy-density flux is constant in Minkowski coordinates, so that in Kruskal coordinates it reads

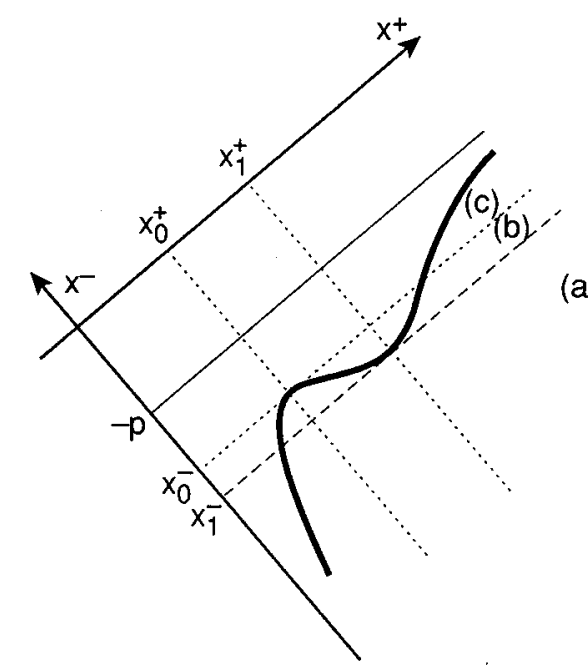

(a)

FIG. 2. Intermediate regime.

$$
T_{++}\left(x^{+}\right)=\frac{\epsilon}{x^{+^{2}}},
$$

whence

$$
\lambda^{2} p=P_{+}\left(x_{1}^{+}\right)=\epsilon\left(\frac{1}{x_{0}^{+}}-\frac{1}{x_{1}^{+}}\right), \quad m=\lambda \epsilon \ln \left(x_{1}^{+} / x_{0}^{+}\right) .
$$

For $x_{1}^{+}$close enough to $x_{0}^{+}$[more precisely, for $x_{1}^{+}<x_{0}^{+} /(1$ $-\kappa / e), \epsilon>\kappa]$ we can have $1+\left(p / x_{0}^{-}\right)>0$ even above the threshold for black hole formation, i.e., with $\epsilon>\kappa$.

\section{THE INTERMEDIATE REGIME}

As mentioned before, Eqs. (3.10) and (3.11) can be continued above the threshold without encountering any singularity up to $p=\left|x_{0}^{-}\right|, p \equiv \lambda^{-2} P_{+}(\infty)$, where the logarithmic singularity appears. We shall call this the intermediate regime, i.e., the case when $p<\left|x_{0}^{-}\right|$and $T_{++}>T_{++}^{\mathrm{cr}}$ for some $x^{+}$, as opposed to the "supercritical" regime where $p$ $>\left|x_{0}^{-}\right|$. The former describes small "Planck-size" black holes, whereas the latter includes macroscopic black holes (the classical picture is approached for $p \gg\left|x_{0}^{-}\right|$).

The geometry is exhibited in Fig. 2 for the case where the incoming energy density is larger than the critical one in the whole range $x_{0}^{+}<x^{+}<x_{1}^{+}$. Region (a) is defined as $x^{-}<x_{1}^{-}$, region (b) as $x_{1}^{-}<x^{-}<x_{0}^{-}$, and region (c) as $x^{-}>x_{0}^{-}$. Figure 2 can be understood as a deformation of Fig. 1. In this process region (ii) and part of (i) of Fig. 1 are superposed into region (b) of Fig. 2. Region (c) is part of region (iii), so that $T_{--}^{(c)}=T_{--}^{(\mathrm{iii})}=0$. It is thus convenient to split the integral (3.10) as

$$
E_{\mathrm{out}}^{(\mathrm{i})}=-\frac{\lambda \kappa p}{x_{0}^{-}}-\lambda \kappa \ln \left(1+\frac{p}{x_{0}^{-}}\right)=E_{\mathrm{out}}^{(\mathrm{a})}+E_{\mathrm{out}}^{(\mathrm{ib})},
$$

where 


$$
\begin{gathered}
E_{\mathrm{out}}^{(\mathrm{a})}=-\lambda \int_{-\infty}^{x_{1}^{-}} d x^{-}\left(x^{-}+p\right) T_{--}^{(\mathrm{i})}=-\frac{\lambda \kappa p}{x_{1}^{-}}-\lambda \kappa \ln \left(1+\frac{p}{x_{1}^{-}}\right), \\
E_{\mathrm{out}}^{(\mathrm{ib})}=-\lambda \int_{x_{1}^{-}}^{x_{0}^{-}} d x^{-}\left(x^{-}+p\right) T_{--}^{(\mathrm{i})} \\
=-\frac{\lambda \kappa p}{x_{0}^{-}}+\frac{\lambda \kappa p}{x_{1}^{-}}-\lambda \kappa \ln \frac{\left(1+p / x_{0}^{-}\right)}{\left(1+p / x_{1}^{-}\right)} .
\end{gathered}
$$

The first integral gives the energy radiated in region (a) of Fig. 2. The second integral contributes to the radiation in region (b). The total energy in region (b) is obtained by adding $E_{\text {out }}^{\text {(ii) }}$ Since now $x_{1}^{-}<x_{0}^{-}$, it is convenient to write this integral in the following way:

$$
\begin{aligned}
E_{\mathrm{out}}^{(\mathrm{ii})} & =-\lambda \int_{x_{1}^{-}}^{x_{0}^{-}} d x^{-}\left(x^{-}+p\right)\left(-T_{--}^{(\mathrm{ii})}\right) \\
& =m+\frac{\lambda \kappa p}{x_{0}^{-}}+\kappa \lambda \ln \left(1+\frac{p}{x_{0}^{-}}\right)
\end{aligned}
$$

so that

$$
\begin{gathered}
E_{\mathrm{out}}^{(\mathrm{b})}=E_{\mathrm{out}}^{(\mathrm{ib})}+E_{\mathrm{out}}^{(\mathrm{ii})}=-\lambda \int_{x_{1}^{-}}^{x_{0}^{-}} d x^{-}\left(x^{-}+p\right) \widetilde{T}_{--}^{(\mathrm{b})}, \\
\widetilde{T}_{--}^{(\mathrm{b})} \equiv T_{--}^{(\mathrm{i})}-T_{--}^{(\mathrm{ii})}, \\
E_{\mathrm{out}}^{(\mathrm{b})}=m+\frac{\lambda \kappa p}{x_{1}^{-}}+\kappa \lambda \ln \left(1+\frac{p}{x_{1}^{-}}\right) .
\end{gathered}
$$

Clearly, $E_{\text {out }}^{(\mathrm{a})}+E_{\text {out }}^{(\mathrm{b})}=m$, so that the whole incoming energy has been radiated [see Eqs. (3.10) and (3.11)]. This means that these black holes evaporate completely.

It should be noticed that in the region (b) (i.e., in the region in causal contact with the apparent horizon) the $T_{--}$arising in the RST formalism does not coincide with the straightforward continuation of the subcritical formulas given by Eq. (4.5). Indeed, in RST the energy-momentum tensor keeps being $T_{--}^{(\mathrm{i})}$ until the geometry is matched with the vacuum. Although in both cases the original energy is completely radiated, the structure of the out-going energydensity flux in the two models is different.

\section{APPARENT HORIZON AS A BOUNDARY}

In the subcritical regime the boundary conditions (3.3) or, equivalently,

$$
\begin{aligned}
& \Omega=\Omega_{\mathrm{cr}}, \\
& \partial_{+} \Omega=0,
\end{aligned}
$$

can be implemented simultaneously on some line. Above the threshold the line defined by Eq. (5.1) is necessarily different from the line defined by Eq. (5.2). The usual choice is to define the boundary line by $\Omega=\Omega_{\mathrm{cr}}$, since it is on this line that the curvature is singular. This leads to the black-hole evolution described in [4] which, although it reproduces the standard Hawking model of gravitational collapse, does not

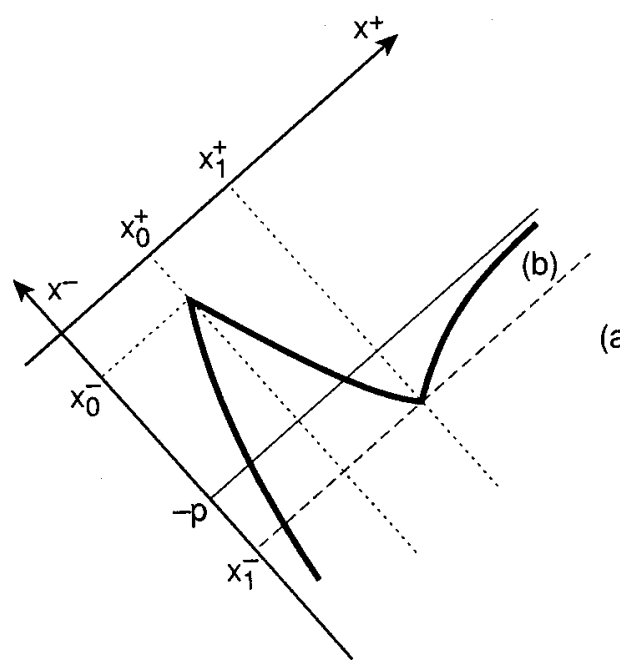

(a)

FIG. 3. Apparent horizon in the supercritical regime.

correspond to the analytic continuation of the subcritical regime. We will now show that the other option, namely imposing boundary conditions at $\partial_{+} \Omega\left(x^{+}, x^{-}\right)=0$ (the apparent horizon), will reproduce the results that were previously obtained by a simple continuation of the subcritical formulas.

Let us assume that the incoming supercritical energydensity flux $T_{++}\left(x^{+}\right)$starts at $x_{0}^{+}$, and it is turned off at a later time $x_{1}^{+}$(a more general situation is discussed in Sec. $\mathrm{VI})$. In region (a) the geometry will be given by Eq. (2.5). The boundary $\partial_{+} \Omega=0$ becomes timelike for $x^{-}>x_{1}^{-}$, and boundary conditions are needed in order to determine the evolution of the geometry in region (b) (see Figs. 2 and 3). Continuity along the line $x_{1}^{-}$requires that

$$
\Omega^{(\mathrm{b})}\left(x^{+}, x^{-}\right)=\Omega^{(\mathrm{a})}\left(x^{+}, x^{-}\right)+F\left(x^{-}\right),
$$

with $F\left(x_{1}^{-}\right)=0$. We need to generalize the expression (3.4) for the case when there is some energy stored in the geometry by the time the boundary becomes timelike. The form of $F\left(x^{-}\right)$in the subcritical regime suggests the choice (the general structure will be clear in Sec. VI)

$F\left(x^{-}\right)=\kappa \ln \left(\frac{x^{-}+\lambda^{-2} P_{+}(u)}{x^{-}+\lambda^{-2} P_{+}\left(x_{1}^{+}\right)}\right)+\lambda^{-1} M(u)-\lambda^{-1} M\left(x_{1}^{+}\right)$,

with $u\left(x^{-}\right)$given by the branch $x_{0}^{+}<u<x_{1}^{+}$of the solution to the equation

$$
-\lambda^{2} u\left[x^{-}+\lambda^{-2} P_{+}(u)\right]=\kappa .
$$

We will find that given the boundary condition (5.3) with Eqs. (5.4) and (5.5), then Eqs. (4.2), (4.5) are reproduced (in particular, this means that this boundary condition conserves energy). The formulas for the outgoing fluxes will be identical to those obtained by direct extrapolation from the subcritical regime.

Let us note that the matching between regions (a) and (b) is smoother than in the case of [4], i.e., there is no outgoing shock wave: 


$$
T_{--}\left(x_{1}^{-}+\epsilon\right)-T_{--}\left(x_{1}^{-}-\epsilon\right)=-\frac{d F}{d x^{-}} \delta\left(x-x_{1}^{-}\right)=0,
$$

since [see Eqs. (5.4) and (5.5)]

$$
\frac{d F}{d x^{-}}=\kappa\left[\frac{1}{x^{-}+\lambda^{-2} P_{+}(u)}-\frac{1}{x^{-}+p}\right]
$$

vanishes at $x^{-}=x_{1}^{-}$.

\section{A. Intermediate regime}

Let us first consider the intermediate regime. In region (c), defined by $-p>x^{-}>x_{0}^{-}$, the geometry is matched with the linear dilaton vacuum: i.e.,

$$
\Omega^{(c)}\left(x^{+}, x^{-}\right)=-\lambda^{2} x^{+}\left(x^{-}+p\right)-\kappa \ln \left[-\lambda^{2} x^{+}\left(x^{-}+p\right)\right] .
$$

The energy-momentum tensor in the different regions are found to be $\left[T_{--}=-\partial_{-}^{2} \Omega+t_{-}\left(x^{-}\right), t_{-}\left(x^{-}\right)=\kappa /\left(x^{-}\right.\right.$ $\left.+p)^{2}\right]$

$$
\begin{gathered}
T_{--}^{(\mathrm{a})}\left(x^{-}\right)=\kappa\left[\frac{1}{\left(x^{-}+p\right)^{2}}-\frac{1}{x^{-2}}\right], \\
T_{--}^{(\mathrm{b})}\left(x^{-}\right)=\lambda^{2} \frac{d u}{d x^{-}}-\frac{\kappa}{x^{-2}}, \quad T_{--}^{(\mathrm{c})}\left(x^{-}\right)=0 .
\end{gathered}
$$

In particular, we note that, since $u^{\prime}=\lambda^{2}\left(\kappa / u^{2}\right.$ $\left.-T_{++}(u)\right)^{-1}<0$ (the flux is above the critical flux), the outgoing flux in region (b) carries negative energy.

Since in region (a) the solution was not modified, one has $T_{--}^{(\mathrm{a})}=T_{--}^{(\mathrm{i})}$ [see Eq. (3.7)]. Now we note the surprising relation [see Eqs. (3.7), (3.8), and (4.5)]

$$
T_{--}^{(\mathrm{b})}=T_{--}^{(\mathrm{i})}-T_{--}^{(\mathrm{ii})} \equiv \widetilde{T}_{--}^{(\mathrm{b})} .
$$

Thus the outgoing energy momentum tensor in this theory with $\partial_{+} \Omega=0$ as a boundary coincides with the extrapolation of the subcritical energy momentum tensor beyond the threshold for black-hole formation, indicating that it is the theory defined with the boundary at the apparent horizon that represents the analytic continuation of the subcritical regime.

\section{B. Supercritical regime}

Let us now proceed by considering the case $p>\left|x_{0}^{-}\right|$(Fig. 3). ${ }^{1}$ The energy-momentum tensor in region (b) can either be obtained by analytic continuation or by using Eqs. (5.3) and (5.4), and it will be given by Eq. (5.9), just as in the intermediate regime. The final $\tau \rightarrow \infty$ geometry for a timelike observer is obtained by taking the limit $x^{+} \rightarrow \infty$ and $x^{-} \rightarrow-p$ in Eqs. (5.3) and (5.4) (recall $\left.2 \lambda \tau=\ln x^{+} /\left|x^{-}+p\right|\right)$ :

\footnotetext{
${ }^{1}$ Note that possible discontinuities in $T_{++}\left(x^{+}\right)$produce discontinuities in the derivative of the curve representing the apparent horizon (such discontinuities can of course be present in all regimes).
}

$$
\begin{aligned}
\left.\Omega^{(\mathrm{b})}\left(x^{+}, x^{-}\right)\right|_{\tau \rightarrow \infty}= & -\lambda^{2} x^{+}\left(x^{-}+p\right) \\
& -\kappa \ln \left[-\lambda^{2} x^{+}\left(x^{-}+p\right)\right]+\frac{m_{f}}{\lambda},
\end{aligned}
$$

$$
m_{f}=M\left(x_{2}^{+}\right)+\lambda \kappa \ln \left(1-\frac{P_{+}\left(x_{2}^{+}\right)}{\lambda^{2} p}\right), \quad x_{2}^{+} \equiv u(-p) .
$$

This is a static geometry with ADM mass equal to $m_{f}$. In the whole of region (b), where $-\lambda^{2} x^{+}\left(x^{-}+p\right)>\kappa$, the logarithmic term can be neglected and the geometry is essentially the same as the classical black-hole geometry. The logarithmic term is only significant close to the line $x^{-}=-p$, where there is a singularity. However, this is beyond the boundary at the apparent horizon.

Let us check that energy is conserved. We now obtain by explicit integration

$$
\begin{aligned}
E_{\mathrm{out}}^{(\mathrm{a})}= & -\lambda \int_{\infty}^{x_{1}^{-}} d x^{-}\left(x^{-}+p\right) T_{--}^{(\mathrm{a})}=-\frac{\lambda \kappa p}{x_{1}^{-}}-\lambda \kappa \ln \left(1+\frac{p}{x_{1}^{-}}\right) \\
E_{\mathrm{out}}^{(\mathrm{b})} & =-\lambda \int_{x_{1}^{-}}^{-p} d x^{-}\left(x^{-}+p\right) T_{--}^{(\mathrm{b})} \\
& =m-M\left(x_{2}^{+}\right)+\frac{\lambda \kappa p}{x_{1}^{-}}+\lambda \kappa \ln \frac{\left(1+p / x_{1}^{-}\right)}{\left[1-P_{+}\left(x_{2}^{+}\right) / \lambda^{2} p\right]},
\end{aligned}
$$

so that

$$
E_{\mathrm{out}}^{(\mathrm{a})}+E_{\mathrm{out}}^{(\mathrm{b})}=m-M\left(x_{2}^{+}\right)+\lambda \kappa \ln \left(\frac{\lambda^{2} x_{2}^{+} p}{\kappa}\right)=m-m_{f},
$$

where we have used the relation $\lambda^{2} x_{2}^{+}\left[p-\lambda^{-2} P_{+}\left(x_{2}^{+}\right)\right]$ $=\kappa$. Thus energy is indeed conserved, and the total radiated energy is positive definite, since

$$
m-M\left(x_{2}^{+}\right)=\lambda \int_{x_{2}^{+}}^{x_{1}^{+}} d x^{+} x^{+} T_{++}>0,
$$

and $\ln \left(\lambda^{2} x_{2}^{+} p / \kappa\right)>0$. Indeed, $\lambda^{2} x_{2}^{+} p / \kappa>\lambda^{2} x_{0}^{+} p / \kappa=p /\left|x_{0}^{-}\right|$, with $p /\left|x_{0}^{-}\right|>1$ in the supercritical regime.

Let us estimate the mass $m_{f}$ of the remaining black hole. For a "macroscopic", black hole, i.e., with $p \gg\left|x_{0}^{-}\right|$, it is clear that $M\left(x_{2}^{+}\right), P_{+}\left(x_{2}^{+}\right)$will not differ much from $M\left(x_{1}^{+}\right), p \equiv P_{+}\left(x_{1}^{+}\right)$, since $x_{2}^{+} \cong x_{1}^{+}$(see Fig. 3 ). We can therefore anticipate that $m_{f} \cong M\left(x_{1}^{+}\right) \equiv m$. This means that very little energy has been radiated and the final black hole will have a mass similar to the total imploding energy. This is very different from the standard picture of Hawking evaporation. To be explicit, let us consider two extreme cases, namely the case of a constant energy-density flux falling in for a long time, and the case of a shock-wave collapse. Using Eqs. (3.12) and (3.13) we find for the former $x_{2}^{+}$ $=x_{1}^{+}(1-\kappa / \epsilon)$, and 
$E_{\mathrm{out}}^{(\mathrm{a})}+E_{\mathrm{out}}^{(\mathrm{b})}=\lambda \kappa \ln \frac{\epsilon}{\kappa}\left(\frac{x_{1}^{+}}{x_{0}^{+}}-1\right)+\lambda(\epsilon-\kappa) \ln \frac{\epsilon}{\epsilon-\kappa}$.

For $x_{1}^{+} / x_{0}^{+} \gg 1$ we get

$$
m-m_{f}=\frac{\kappa}{\epsilon} m
$$

Since $p \gg\left|x_{0}^{-}\right|$implies $\epsilon \gg \kappa$, this is a small quantity. The total radiated energy in the opposite limit of a shock wave can be found by using Eq. (5.15) and the fact that, for a shock wave, $p=m / \lambda^{3} x_{0}^{+}$. This gives

$$
m_{f}=m-\lambda \kappa \ln \frac{m}{\lambda \kappa} .
$$

While the radiated energy logarithmically increases with $m$, the ratio $m_{f} / m \rightarrow 1$ as $m \rightarrow \infty$.

\section{Outgoing energies}

The energies radiated in region (i) of Fig. 1 and in region (a) of Figs. 2 and 3 are positive definite, since they are the integral of a positive-definite quantity [see Eq. (3.7)]. We have also seen in the previous subsection that the total radiated energy is positive definite. In the subcritical regime-as mentioned in Sec. III-the energy in region (ii) can be positive or negative, depending on the characteristics of the incoming flux. This will be clear from the examples that we give below. As pointed out after Eq. (5.9), the energy $E_{\text {out }}^{(\mathrm{b})}$ is negative definite, being the integral of a negative-definite quantity [see Eq. (5.9)]. Here we show that this negative energy is of the order of the Planck mass, i.e., smaller than $O(\lambda \kappa)$. This characteristic is present in the RST model as well, where negative energy is carried out by a shock wave (the "thunderpop"') at the endpoint of black-hole evaporation. As shown below, here the analogue endpoint wave is smeared in a Planck time.

We start by considering the particular example of the constant incoming flux given by Eq. (3.12). Using Eqs. (3.13), (3.11), (4.6), and (5.14) one finds the following expressions.

Subcritical regime $[a, y \in(0,1)]$ :

$E_{\mathrm{out}}^{(\mathrm{ii})}=\lambda \kappa[-a \ln y-a(1-y)+\ln (1-a+y a)]$.

Intermediate regime $\left[a \in(1, \infty), y \in\left(y_{0}, 1\right)\right]$ :

$E_{\mathrm{out}}^{(\mathrm{b})}=\lambda \kappa\left[-a \ln y-\left(1+b^{-1}\right)^{-1}-\ln (1+b)\right]$.

Supercritical regime $\left[a \in(1, \infty), y \in\left(0, y_{0}\right)\right]$ :

$E_{\mathrm{out}}^{(\mathrm{b})}=\lambda \kappa\left[(1-a) \ln \left(1-a^{-1}\right)-\left(1+b^{-1}\right)^{-1}-\ln \left(1+b^{-1}\right)\right]$,

where

$$
a \equiv \frac{\epsilon}{\kappa}, \quad y \equiv \frac{x_{0}^{+}}{x_{1}^{+}}, \quad y_{0}=1-a^{-1}, \quad b \equiv a\left(y^{-1}-1\right)
$$

It can be easily seen that the minimum value of $E_{\text {out }}^{(\mathrm{ii})}, E_{\text {out }}^{(\mathrm{b})}$ given by Eqs. (5.18) $-(5.20)$ is $-\lambda \kappa$, and it occurs at the point $y=0$ and $a=1$ (corresponding to an incoming flux equal to the critical flux lasting forever). Thus

$$
E_{\text {out }}^{(\mathrm{b})} \geqslant-\lambda \kappa \text {. }
$$

This is essentially the same bound as appears in the RST model. Although we have proved Eq. (5.21) for a constant incoming flux, a similar bound can be obtained in the general case. Consider the general expression for $E_{\text {out }}^{(\mathrm{b})}$ in the supercritical regime (which includes the case of macroscopic black holes). It is convenient to write Eq. (5.14) in the form

$$
\begin{aligned}
E_{\mathrm{out}}^{(\mathrm{b})}= & m-M\left(x_{2}^{+}\right)+\frac{\lambda \kappa p}{x_{1}^{-}}-\lambda \kappa \ln \left(1+\frac{\kappa}{\lambda^{2} p x_{1}^{+}}\right) \\
& -\lambda \kappa \ln \left(x_{1}^{+} / x_{2}^{+}\right) .
\end{aligned}
$$

From the inequalities

$$
\begin{aligned}
m-M\left(x_{2}^{+}\right) & =\lambda \int_{x_{2}^{+}}^{x_{1}^{+}} d x^{+} x^{+} T_{++}>\lambda \int_{x_{2}^{+}}^{x_{1}^{+}} d x^{+} x^{+} T_{++}^{\mathrm{cr}} \\
& =\lambda \kappa \ln \left(x_{1}^{+} / x_{2}^{+}\right),
\end{aligned}
$$

$\lambda \kappa p / x_{1}^{-}>-\lambda \kappa$, and

$$
\begin{aligned}
-\lambda \kappa \ln \left(1+\frac{\kappa}{\lambda^{2} p x_{1}^{+}}\right) & >-\lambda \kappa \ln \left(1+\frac{\kappa}{\lambda^{2} p x_{0}^{+}}\right) \\
& =-\lambda \kappa \ln \left(1-\frac{x_{0}^{-}}{p}\right)>-\lambda \kappa \ln 2
\end{aligned}
$$

we obtain

$$
E_{\text {out }}^{(\mathrm{b})} \geqslant-\lambda \kappa-\lambda \kappa \ln 2 .
$$

Next, let us estimate the time interval of the negative energy emission. For simplicity we will consider the case of a constant incoming flux. In Minkowski coordinates $\sigma^{ \pm}$the energy momentum tensor (5.9) takes the form

$$
\begin{gathered}
T_{--}^{(\mathrm{b})}=-\kappa\left[\frac{(\epsilon / \kappa-1)}{\left[(\epsilon / \kappa) e^{\lambda \tau^{-}}-1\right]^{2}}+\frac{1}{\left[1+\left(\lambda^{2} p x_{1}^{+} / \kappa\right) e^{\lambda \tau-}\right]^{2}}\right] \\
\lambda \tau^{-} \equiv \lambda \sigma^{-}-\ln \left(\lambda x_{1}^{+} / \kappa\right)
\end{gathered}
$$

The shifted Minkowski time $\tau^{-}$is such that it starts at 0 when the negative energy emission begins. The second term in Eq. (5.23) is always negligible with respect to the first one. Since $\epsilon / \kappa>1, T_{--}^{(\mathrm{b})}$ is an exponentially decreasing function, with a damping time interval of order $\Delta \tau^{-}=\frac{1}{2} \lambda^{-1}$, i.e., a "Planckian" interval of time [more precisely, $\Delta \tau^{-}$ $\left.=\frac{1}{2} \lambda^{-1}(1-\kappa / \epsilon)<\frac{1}{2} \lambda^{-1}\right]$. 


\section{MORE GENERAL DISTRIBUTIONS OF INCOMING MATTER}

To complete the physical picture, let us also give the geometry in region (b) in the case when the incoming energydensity flux does not stop at $x_{1}^{+}$. Let us assume that $T_{++}\left(x^{+}\right)$is a smooth function of $x^{+}$for all $x^{+}>x_{0}^{+}$, and define $x_{1}^{+}$as the point at which $T_{++}\left(x^{+}\right)$becomes less than the critical flux, so that the apparent horizon becomes timelike after this point. Continuity along the line $x_{1}^{-}$requires that

$$
\Omega^{(\mathrm{b})}\left(x^{+}, x^{-}\right)=\Omega^{(\mathrm{a})}\left(x^{+}, x^{-}\right)+F\left(x^{-}\right),
$$

with $F\left(x_{1}^{-}\right)=0$ and $\Omega^{(a)}$ as given by Eq. (2.5). The expression that generalizes Eqs. (3.4) and (5.4) is

$F\left(x^{-}\right)=\kappa \ln \left(\frac{x^{-}+\lambda^{-2} P_{+}(u)}{x^{-}+\lambda^{-2} P_{+}\left(\hat{x}^{+}\right)}\right)+\lambda^{-1} M(u)-\lambda^{-1} M\left(\hat{x}^{+}\right)$,

with $u\left(x^{-}\right)$given as before by the branch $x_{0}^{+}<x^{+}<x_{1}^{+}$of the solution $u=x^{+}\left(x^{-}\right)$to the equation

$$
-\lambda^{2} x^{+}\left[x^{-}+\lambda^{-2} P_{+}\left(x^{+}\right)\right]=\kappa,
$$

and $\hat{x}^{+}\left(x^{-}\right)$given by the upper branch $x^{+}>x_{1}^{+}$. As in the case of Sec. V, there is no shock-wave discontinuity in going from region (a) to region (b), since $F^{\prime}\left(x_{1}^{-}\right)=0$ [interestingly, $\lambda^{-2} F^{\prime}\left(x^{-}\right)=\hat{x}^{+}-u$, i.e., the distance between the two points of the apparent horizon corresponding to a given $\left.x^{-}\right]$.

The energy-momentum tensor in region (a) is as in Eq. (5.8) [since the solution is the same in this region), and in region (b) one finds $\left[p \equiv \lambda^{-2} P_{+}(\infty)\right]$

$$
\begin{aligned}
& T_{--}^{(\mathrm{b})}\left(x^{-}\right)=-\partial_{-}^{2} \Omega+\frac{\kappa}{\left(x^{-}+p\right)^{2}} \\
&=\lambda^{2} \frac{d u}{d x^{-}}-\lambda^{2} \frac{d \hat{x}^{+}}{d x^{-}}-\frac{\kappa}{x^{-2}}+\frac{\kappa}{\left(x^{-}+p\right)^{2}}, \\
& T_{--}^{(\mathrm{c})}\left(x^{-}\right)=0 .
\end{aligned}
$$

This is essentially the energy-density flux of Eq. (5.9) plus an additional (positive energy) contribution of the form (3.8) representing reflection of the $T_{++}\left(x^{+}\right), x^{+}>x_{1}^{+}$on the timelike apparent horizon. The total mass of the final black-hole geometry will not vary too much by bombarding it with subcritical energy density. Indeed, using Eqs. (6.1) and (6.2) we find that the final geometry at $x^{+} \rightarrow \infty, x^{-} \rightarrow-p$ is given by

$$
\begin{gathered}
\Omega^{(\mathrm{b})}\left(x^{+}, x^{-}\right)=-\lambda^{2} x^{+}\left(x^{-}+p\right)-\kappa \ln \left[-\lambda^{2} x^{+}\left(x^{-}+p\right)\right] \\
+\frac{m_{f}}{\lambda}, \\
m_{f}=M\left(x_{2}^{+}\right)+\lambda \kappa \ln \left(1-\frac{P_{+}\left(x_{2}^{+}\right)}{\lambda^{2} p}\right), \quad x_{2}^{+} \equiv u(-p) .
\end{gathered}
$$

This is approximately the same static black hole as in the previous case, Eq. (5.11), except that now $p$ is slightly different [since the energy-density flux for $x^{+}>x_{1}^{+}$is subcritical, it can be easily seen that $\left.P_{+}(\infty)-P_{+}\left(x_{1}^{+}\right)<\kappa / x_{1}^{+}\right]$. This difference produces only a tiny (Planck-scale) increase in the final mass $m_{f}$ with respect to Eq. (5.11).

At first sight, the fact that, for $x^{+}>x_{1}^{+}$, low-energy density matter reflects on the apparent horizon may seem strange. However, it must be stressed that this is a quantum effect, since only a subcritical energy-density flux would reflect. If, after $x_{1}^{+}$, supercritical matter is sent in, the apparent horizon will become spacelike and all but a Planckian bit of energy will be eaten by the black hole, increasing its size in accordance with the total energy of the additional matter.

\section{OUTLOOK AND DISCUSSION}

Here we have explored the theory which results from analytically continuing the subcritical regime above the threshold of black-hole formation. In the corresponding semiclassical theory, quantum effects appear in various ways, but the net result is that only small alterations over a classical picture appear. Let us summarize the picture.

(1) Collapsing macroscopic matter (i.e., with incoming energy-momentum tensor far above the threshold for black hole formation) forms stable black holes with masses of the same order as the total imploding energy plus minor emission. This involves Hawking radiation at early times and a subsequent burst with tiny energy (of order of the Planck mass).

(2) If the infalling matter has densities not much larger than the critical one, the situation looks similar to the conventional Hawking picture. This is the intermediate regime where a small black hole is formed and evaporates completely.

(3) Infalling subcritical matter over an already formed black hole will be reflected from the apparent horizon with a small accompanying evaporation.

(4) Macroscopic matter falling over a black hole will simply increase its mass and give rise to a limited emission, as in (1).

The bursts have negative energies of order $\kappa \lambda$ and last a short Planckian time $\lambda^{-1}$. A similar feature appears in the RST model, where the matching with the vacuum is made at the price of a shock-wave discontinuity; this shock wave (the "thunderpop") carries out "Planckian" negative energy. In the present model, the different regions are smoothly matched. In a sense, the shock wave is smeared-out in a Planckian interval of time.

Why is the final geometry stable? The vanishing of the energy-momentum tensor at infinity requires-just as when the Boulware vacuum is adopted [6] - a substantial modification of the geometry near the line $x^{-}=-p$. As we have seen in Sec. V B, this is exactly what is happening. In the allowed space-time region $-\lambda^{2} x^{+}\left(x^{-}+p\right)>\kappa$ the geometry is essentially the same as the classical black-hole geometry. Only at $-\lambda^{2} x^{+}\left(x^{-}+p\right) \ll e^{-m / \lambda \kappa}$ is the geometry significantly modified, but this lies beyond the boundary. 
The model agrees with the Hawking theory in the region that is not in causal contact with the apparent horizon [called region (a) in Fig. 3]. Beyond this point [region (b)], a quantum theory of gravity is required in order to predict the outgoing spectrum, since outgoing modes have Planck frequencies at the moment they arise from the vicinity of the horizon (i.e., about one Planck proper distance from the horizon; see Refs. [1,7-9]). Lacking a microscopic theory, some extra phenomenological input is needed, and several possibilities have been discussed [8,10-12]. In the context of this twodimensional model, this is naturally realized in two scenarios. The first one, described in RST, is based on a quantum field theory with a boundary at $\Omega=\Omega_{\mathrm{cr}}$ (the singularity); the other, described here, follows from analytic continuation and implies a boundary at $\partial_{+} \Omega=0$ (the apparent horizon). For the former, the physics above the threshold reproduces the Hawking model of gravitational collapse, and thus leads to information loss. But, as we have seen, this physics is not analytically connected to the subcritical regime: an $S$ matrix constructed on the basis of the subcritical theory would not describe this conventional approach. ${ }^{2}$

In Refs. $[1,11]$ the unitarity property of the $S$ matrix was used to constrain the number of fundamental degrees of freedom in quantum gravity within a given volume (see also $[14,12])$. But unitarity is not the only implication of having an $S$ matrix: an $S$ matrix also requires that the physics above the thresholds is described by the same (analytically continued) formulas that govern the physics below the thresholds. Surprisingly, the consequence is that black holes stop evaporating. It is important to understand the physics that may give rise to the different picture. The difference stems from the quanta that a distant observer in our model is absent, while the observer of the conventional approach would be interpreted as being originated from the small trapped region (of Planckian proper length) which is in the causal past of null infinity, i.e., in between the receding apparent horizon and the null line $x^{-}=-p$. Those quanta would have experienced a tremendous red shift from trans-Planckian frequencies of order $\lambda e^{\text {const } M / \lambda}$, so their inhibition seems in line with the ultraviolet softening expected from quantum gravity. The boundary has thus the same effect as imposing a cutoff at a Planck-scale frequency, which leads to a termination of the Hawking process [7].

As pointed out by 't Hooft [1], the inertial infalling observer cannot be used to argue that outgoing radiation at sub-Planckian distances from the horizon is not affected by quantum gravity effects, since this observer sees no Hawking radiation at all. There is no contradiction, since it is not possible for the ingoing observer to communicate the result of any physical measurement to the outside world. The description of the physics in the infalling frame is different. To an inertial infalling observer, strong quantum gravity effects only occur near the singularity, so no substantial change with respect to the classical Einstein physics is expected in the horizon region. In the present context, this is consistent with

\footnotetext{
${ }^{2} \mathrm{We}$ stress that we have continued the expressions for the energymomentum tensor, since a satisfactory $S$-matrix formalism in 1 +1 dimensions is, unfortunately, still lacking (despite some interesting attempts [13]).
}

the fact that the analytic continuation we investigate can only be done in the external world; for the infalling observer there are no Hawking particles, so no in-out $S$ matrix to be analytically continued. In passing, we would like to stress that no wall prevents a macroscopic infalling object from entering into the black hole. As a macroscopic object is falling in, the apparent horizon expands and the object always remains inside the black hole. It is only the small emitted radiation that is effectively described as if there was a boundary at the apparent horizon.

The boundary condition at the apparent horizon is not equivalent to the 't Hooft $S$-matrix approach $[1,11]$ or the stretched horizon of Susskind et al. $[15,8],{ }^{3}$ where an effective dynamical boundary is proposed with the task of "transfering" the quantum mechanical information of the incoming matter to the outgoing modes. In those approaches, the discrepancy in the descriptions of distant and freely infalling observers has to be explained in terms of a rather strong notion of complementarity, where quantum gravity effects must be such that they "destroy" the infalling object, extract its quantum mechanical information, and transfer it to the outgoing modes (which must happen even before the object reaches the apparent horizon). This is not the case in the present approach: both ingoing and outside observers agree that the bulk of matter and its information remain in the hole (i.e., beyond the event horizon). There is no need of duplication of the information and no conflict with causality. Here we only make use of 't Hooft's arguments insofar as the emission of trans-Planckian modes must be affected by quantum gravity effects. We are just exploiting the fact that the quantum theoretical description which is appropriate to an outside observer does not need to be extrapolated up to the singularity; analyticity indeed dictates that simple Dirichlet- or Neumann-type boundary conditions must be imposed to the quantum fields at the apparent horizon (which amounts to exclude the region where the contours $r=e^{\phi}$ $=$ const are spacelike).

The dynamics could be quite different in the $(3+1)$-dimensional physics, where it is possible to have classical scattering without black hole formation (e.g., in terms of the impact parameter). In $1+1$ dimensions there is no classical scattering without black holes; the threshold is a pure quantum effect (a model in $3+1$ dimensions is investigated in [17]). The results of this two-dimensional model, provide, however, a simple and concrete example of how the requirement of analytic continuation from a subcritical regime-inevitable in an $S$-matrix approach-may shed light on black-hole behavior in a theory where there is no loss of quantum coherence.

\section{ACKNOWLEDGMENTS}

This work was partially supported by EC Contract No. ERBFMRXCT960090.

\footnotetext{
${ }^{3}$ The apparent horizon always lies inside the stretched horizon and it coincides with it after the incoming flux terminates [16] - in the present model the stretched horizon is just given by $-\lambda^{2} x^{+}\left[x^{-}\right.$ $\left.+\lambda^{-2} P_{+}(\infty)\right]=\kappa$.
} 
[1] G. 't Hooft, Nucl. Phys. B256, 727 (1985); B335, 138 (1990).

[2] D. Amati, M. Ciafaloni, and G. Veneziano, Int. J. Mod. Phys. A 3, 1615 (1988); Nucl. Phys. B403, 707 (1993).

[3] S. Hawking, Commun. Math. Phys. 43, 199 (1975).

[4] J. G. Russo, L. Susskind, and L. Thorlacius, Phys. Rev. D 46, 3444 (1992); 47, 533 (1993).

[5] S. Giddings, Phys. Rev. D 46, 1347 (1992).

[6] D. G. Boulware, Phys. Rev. D 11, 1404 (1975).

[7] T. Jacobson, Phys. Rev. D 44, 1731 (1991).

[8] L. Susskind, L. Thorlacius, and J. Uglum, Phys. Rev. D 48, 3743 (1993).

[9] Y. Kiem, H. Verlinde, and E. Verlinde, Phys. Rev. D 52, 7053 (1995).

[10] L. Susskind, Phys. Rev. Lett. 71, 2367 (1993).
[11] C. Stephens, G. 't Hooft, and B. F. Whiting, Class. Quantum Grav. 11, 621 (1994).

[12] L. Susskind, J. Math. Phys. (N.Y.) 36, 6377 (1995).

[13] H. Verlinde and E. Verlinde, Nucl. Phys. B406, 43 (1993); K. Schoutens, H. Verlinde, and E. Verlinde, Phys. Rev. D 48, 2670 (1993).

[14] G. 't Hooft, Phys. Scr. T36, 247 (1991); in Salamfestschrift, Proceedings of the Conference, Trieste, Italy, 1993, edited by A. Ali et al. (World Scientific, Singapore, 1993), gr-qc/ 9310026.

[15] K. Thorne, R. Price, and D. MacDonald, Black Holes: The Membrane Paradigm (Yale University Press, New Haven, CT, 1986).

[16] J. G. Russo, Phys. Lett. B 359, 69 (1995).

[17] J. G. Russo, Phys. Rev. D 55, 871 (1997). 